\title{
Desenvolvimento de equipamento de auxílio à visão subnormal
}

\author{
Development of a low vision aid device
}

\section{Fernanda Alves da Silva Bonatti*}

Trabalho realizado na Faculdade de Arquitetura e Urbanismo da USP e no Departamento de Oftalmologia da Faculdade de Medicina da Universidade de São Paulo - USP.

* Médica especialista em Otorrinolaringologia pelo Hospital das Clínicas da Faculdade de Medicina da Universidade de São Paulo - USP - São Paulo (SP) - Brasil. Arquiteta graduada pela Faculdade de Arquitetura e Urbanismo da USP - São Paulo (SP) - Brasil.

Endereço para correspondência: Fernanda Alves da Silva Bonatti. Rua Madre Mazarello, 199 - São Paulo (SP) CEP 05454-040

E-mail: bonatti@uol.com.br

A autora não tem interesse comercial nos produtos mencionados neste estudo.

Recebido para publicação em 23.06.2005

Versão revisada recebida em 11.10.2005

Aprovação em 26.10.2005

Nota Editorial: Depois de concluída a análise do artigo sob sigilo editorial e com a anuência da Dra. Luciene Chaves Fernandes sobre a divulgação de seu nome como revisora, agradecemos sua participação neste processo.

\begin{tabular}{|l|}
\hline RESUMO \\
\hline Objetivo: Design de equipamento de uso para perto para portador de \\
visão subnormal. Métodos: Verificar os principais produtos de auxílio à \\
visão subnormal, identificando problemas que o usuário possa ter ao \\
usá-los e por meio de possíveis intervenções do design fazer um projeto \\
que possa contemplar suas necessidades. Resultados: Foi desenvolvido \\
um protótipo compreendido por prancha de leitura em que pode ser \\
conectado o cabo de uma lupa, que se movimenta horizontal e ver- \\
ticalmente possibilitando o acompanhamento de uma linha do texto. \\
Conclusão: O equipamento desenvolvido vem de encontro às necessi- \\
dades visuais e de manuseio de portadores de visão subnormal para \\
perto, incorporando facilidades para a leitura e escrita.
\end{tabular}

Descritores: Baixa visão; Equipamentos e provisões; Desenho de equipamento; Leitura

\section{INTRODUÇ̃̃̃O}

A orientação dos professores para o Trabalho Final de Graduação (TFG) desenvolvido pelos alunos no último semestre da Faculdade de Arquitetura e Urbanismo da Universidade de São Paulo (FAUUSP) tem sido direcionada para projetos de interesse público. Sob a orientação da Prof ${ }^{\underline{a}}$. Dr ${ }^{\underline{a}}$. Maria Cecília Loschiavo dos Santos, ao escolher o tema do TFG, enquanto aluna do último semestre da FAUUSP no segundo semestre de 2004, fiz a opção de também tirar proveito de minha outra formação, pois sou médica, formada na Faculdade de Medicina da Universidade de São Paulo em 1984. Julguei, portanto, ter conhecimentos que poderiam se aliar para o desenvolvimento de um projeto em que essas duas formações fossem necessárias.

Pensando na importância social do design para a saúde, particularmente o design dos equipamentos médicos, iniciei uma pesquisa para definir uma área de atuação. A problemática da visão subnormal vem sendo atendida com equipamentos que, do ponto de vista do design, não são satisfatórios, especialmente no Brasil. É extremamente reduzido o investimento nessa área, o que justificou o estudo em questão.

Desde a Antigüidade aparecem relatos sobre a dificuldade de visão. Os primeiros registros históricos sobre a existência de lentes rudimentares foram escritos na China pelo filósofo Confúcio, em 500 a.C. Na época de Aristóteles (filósofo grego que viveu de 384 a.C. a 322 a.C.) o homem produzia pedras cortadas de maneira a possibilitar a sua utilização como instrumento óptico. Foi somente na Idade Média que os monges começaram a desenvolver a chamada "pedra de leitura", segundo as teorias mais aperfeiçoadas do matemático árabe Alhazen, que viveu em Basra, aproximadamente no ano 1000 d.C. Esta pedra funcionava como uma lupa primitiva 
que aumentava o tamanho das letras e era composta basicamente de cristal de quartzo hialino ou de pedras semipreciosas que tinham lapidação e polimento ${ }^{(1)}$. Em 1270 d.C. Marco Polo encontrou chineses idosos utilizando óculos para a leitura. Existem indicações que foi em Veneza, Itália, vizinha a Murano, que dominava o comércio de vidros, que, no final do século XIII, surgiu uma armação com um par de lentes para se colocar na frente dos dois olhos, com a finalidade de leitura, mas o primeiro auxílio para a correção óptica foi atribuído a René Descartes em 1637 d.C., que descreveu um cone de vidro com uma superfície frontal plana e uma superfície posterior côncava ${ }^{(2)}$.

Uma visão normal é descrita, pelos oftalmologistas, como 20/20, segundo o padrão Snellen (o que significa que a pessoa vê a 20 pés o que a média da população vê a 20 pés). De acordo com a 10 ${ }^{\underline{a}}$. Revisão da Classificação Estatística Internacional de Doenças e Problemas Relacionados à Saúde, Organização Mundial de Saúde, 1993, uma pessoa é considerada como portadora de baixa visão se a sua acuidade visual com correção óptica no melhor olho se encontra entre 20/70 e 20/400 (categorias 1 e 2 da graduação de deficiência visual) e é considerada cega se esses valores estiverem abaixo de 20/400 (categorias 3, 4 e 5) $)^{(3)}$. Em 1992 a OMS propôs uma definição funcional para a baixa visão: "uma pessoa com baixa visão é aquela que tem prejuízo da função visual mesmo depois do tratamento e/ou correção refrativa habitual, e tem uma acuidade visual de menos que 20/70 até a percepção de luz, ou um campo visual menor que $10^{\circ}$ a partir do ponto de fixação, mas utiliza, ou está potencialmente apta a utilizar, a visão para o planejamento e/ou execução de tarefas"(4).

Em 1990 a OMS estimou a existência de 110 milhões de pessoas com deficiências visuais graves no mundo; destes, 38 milhões seriam cegos e 72 milhões possuiriam baixa visão ou visão subnormal. A extrapolação subseqüente desses dados para a população de 1996 ampliou-os para 45 milhões de cegos e 135 milhões de indivíduos com baixa visão. A OMS prevê o acelerado crescimento da cegueira de um a dois milhões de casos por ano, provavelmente dobrando o número total de casos ao redor do ano 2020, a não ser que sejam disponibilizados recursos suficientes para a prevenção ${ }^{(5)}$. No Brasil, existem hoje 16,5 milhões de deficientes visuais. Eles fazem parte do grupo mais numeroso entre os portadores de algum tipo de deficiência. Representam $48 \%$ do total, dos quais 160 mil deles nada enxergam e os demais apresentam algum tipo de perda visual (dados do último Censo Demográfico do IBGE, de $2000)^{(6)}$. A estimativa da prevalência de cegueira no Brasil sofre variações de acordo com os diferentes níveis socioeconômicos do país: 0,25\% em locais semelhantes a países desenvolvidos e $0,75 \%$ em áreas mais pobres economicamente ${ }^{(5)}$.

As causas da baixa visão podem ser divididas em dois grandes grupos: infância e idade adulta. As crianças correspondem a apenas cerca de $4 \%$ do total; nelas, a baixa visão pode ser causada pela coriorretinite macular (perda da visão central), catarata congênita, retinopatia da prematuridade e doenças degenerativas da retina, as distrofias retinianas, se- gundo a médica Maria Aparecida Onuki Haddad, colaboradora do Serviço de Visão Subnormal do Hospital das Clínicas da Faculdade de Medicina da USP (HCFMUSP). "Em idosos, a principal causa é a degeneração macular relacionada à idade, que afeta a visão central, seguida da retinopatia diabética. A terceira causa mais comum é o glaucoma, que leva à perda da visão periférica", explica o Prof. Dr. Marcos Sampaio, coordenador do Serviço de Visão Subnormal do HCFMUSP.

Oitenta por cento dos casos de baixa visão são de pessoas com mais de 60 anos. Estatísticas feitas na Europa e nos Estados Unidos mostram que a condição afeta $6 \%$ da população com 65 anos de idade, número que sobe para $20 \%$ da população a partir dos $85 \operatorname{anos}^{(7)}$. Diversas pesquisas realizadas recentemente registram um forte aumento no número de pessoas com idade superior a 60 anos no Brasil. As estimativas apontam que a proporção de idosos no país representará $10 \%$ da população total até o ano de $2010^{(8)}$.

A baixa visão é relatada como o terceiro mais importante problema crônico do idoso, após as artrites e as cardiopatias $^{(9)}$. Infelizmente não podem ser corrigidas através de cirurgias, medicações ou lentes corretivas habituais. Para aqueles cuja baixa visão está estabilizada e não se encontram satisfeitos com a sua qualidade, existem objetos que podem auxiliar e melhorar a qualidade visual para que possam exercer as atividades mais requisitadas por essas pessoas, que são geralmente a leitura e assistir à televisão.

O Prof. Dr. Marcos W. Sampaio ${ }^{(10)}$, citando Colenbrander ${ }^{(11)}$, escreve que, de acordo com as classes de resposta visual propostas na $9^{\underline{a}}$ Revisão da Classificação Internacional das Doenças (1978), existe uma correlação entre valores de acuidade visual e o emprego de auxílios para o paciente. Estão indicados, assim, equipamentos para ampliação da imagem na visão subnormal moderada (acuidade visual de 20/80 a 20/160) e na visão subnormal grave (de 20/200 a 20/400). Na visão subnormal profunda (de 20/500 a 20/1000) os auxílios indicados são os recursos audíveis e o braile.

$\mathrm{O}$ aumento da imagem se daria com lentes convergentes ou sistema de lentes cujo princípio seria a ampliação por diminuição da distância relativa, colocando-se o objeto de leitura aproximadamente na distância focal da lente.

Lembrar que: distância focal = 1/dioptria, portanto em uma lente de 20 dioptrias positivas o foco está a $0,05 \mathrm{~m}$ ou $5 \mathrm{~cm}$.

Simplificadamente pode-se dizer que a quantidade do aumento proporcionado por uma lente positiva é dado pelo número de dioptrias dividido por 4. Assim, uma lente de 20 dioptrias aumenta em 5 vezes uma imagem.

Porém, quanto maior o aumento proporcionado pela lente, fica mais difícil focalizar, e menor é o campo visual obtido ("o quanto se consegue ver, horizontal e verticalmente") e quanto menor a distância de trabalho, maior é a limitação de tarefas.

Os recursos auxiliadores na baixa visão partem do mesmo princípio: a magnificação ou ampliação da imagem. Eles podem ser divididos em 3 grandes grupos $^{(7)}$ :

a) para perto: lupas manuais, lupas de apoio, óculos com adições especiais e telemicroscópios. 
b) para longe: os sistemas telescópicos, que também são indicados para aumentar a imagem para perto. Podem ser monoculares ou binoculares, exigindo boa coordenação motora do paciente para ajustar o foco; não são esteticamente agradáveis, apresentam custo elevado e reduzem o campo visual.

c) sistemas de videomagnificação: também conhecidos como CCTV (closed circuit television), usados para a ampliação da imagem projetada através da tela da televisão. São aparelhos que se utilizam de projeções eletrônicas, requeridos pelos pacientes que já não podem se beneficiar dos outros sistemas, o que corresponde a uma minoria das pessoas com visão subnormal.

\section{OBJETIVOS}

Os principais objetivos que nortearam esse trabalho foram: mapear o estado da arte dos equipamentos para visão subnormal; identificar necessidades que possam não estar sendo atendidas pelos principais equipamentos atualmente existentes; identificar possíveis intervenções do design que possam contribuir para atender as necessidades anteriormente verificadas; investir no design de um equipamento que tenha qualidades estéticas que contribuam para desenvolver um estado de ânimo mais favorável nos deficientes visuais, melhorando a sua qualidade de vida; projetar procurando não incentivar ou até minimizar os preconceitos ligados às deficiências.

\section{MÉTODOS}

\section{Pesquisa de produtos de auxílio à visão subnormal}

Foram levantadas referências bibliográficas a partir de material publicado em livros, revistas e sítios de Internet. Foram realizadas visitas a revendedores que comercializam tais produtos para poder-se realizar uma avaliação estética e funcional dos mesmos.

A partir dessa análise, foram inferidas algumas necessidades que não eram atendidas pelos equipamentos, tais como o cansaço muscular ao longo de um tempo de leitura que uma pessoa teria ao segurar as lupas manuais ou as lupas de apoio para a leitura e a dificuldade para escrever ao utilizar-se as lupas manuais ou de apoio.

Obteve-se então um programa de necessidades.

\section{Definição do tipo de equipamento a ser projetado e do perfil dos usuários}

O programa de necessidades estabeleceu o projeto de um produto que integrasse a função de "segurar" a lupa para o paciente quando ele sentisse cansaço muscular com a função de facilitar o acompanhamento da linha horizontal de leitura.

$\mathrm{O}$ equipamento foi projetado visando à ampliação da imagem na visão de perto, voltado para a faixa de visão subnormal moderada (acuidade visual de 20/80 a 20/160) e grave (de 20/200 a 20/400).

Foram utilizados:

- lente de aumento asférica de +15 dioptrias procedente da empresa SOLA, com 62 mm de diâmetro,

- placa de PVC (policloreto de vinila), para a prancha de leitura e seu apoio na região posterior,

- perfis de alumínio em U da Alcoa, código PU-021 com medidas horizontais e verticais simétricas e iguais a $15,87 \mathrm{~mm}$ e espessura de $2,38 \mathrm{~mm}$,

- tarugo de nylon de $60 \mathrm{~mm}$ de diâmetro para confecção das peças deslizantes,

- braço com um sistema de eixos, encontrado pronto no mercado,

- "luva rosca" de PVC, de duas polegadas marca Tigre,

- botões plásticos com parafuso acoplado, de mercado,

- dobradiça, de comprimento $9 \mathrm{~cm}$, marca Papaiz,

- parafusos de 3/16 polegadas de diâmetro,

- lonas impermeáveis cinza e vermelha, tecido de veludo, alças reguláveis, zíper, para a confecção de bolsa de armazenamento de protótipo,

Local: LAME, Laboratório de Modelos e Ensaios da FAUUSP no período de 10 a 14 de janeiro de 2005.

\section{RESULTADOS}

Segurar uma lupa para a leitura é uma atividade cansativa, tanto mais quanto mais idoso for o paciente, ou apresentar dificuldades motoras associadas. É difícil escrever segurando uma lupa em uma das mãos. As pessoas podem apresentar grande dificuldade em segurar a lupa enquanto lêem, pois é preciso ajustar a distância focal a todo o momento. Ao utilizar uma lupa de apoio o foco estará fixo, mas eles também se cansam porque é necessário segurar a lupa e deslizá-la pelo texto. Os idosos se cansam com facilidade e tal dificuldade se acentua em pessoas com problemas motores associados, como por exemplo, na doença de Parkinson.

Assim, ao inferir esses problemas, foi desenvolvido o projeto de um produto que possa apoiar uma lupa e permitir o seu deslizamento enquanto o paciente lê ou escreve. Ao se cansar, sua lupa não cairá, pois ela estará fixa ao equipamento.

Pesquisando os equipamentos não ópticos de auxílio à visão subnormal, encontrei pranchas de leitura reguláveis para diversas inclinações, sem lupas acopladas, que permitem que o paciente apóie o seu material de leitura ou escrita, porém, ao utilizar uma lupa, ela terá que ser segurada o tempo todo. O material utilizado nessas pranchas é a madeira (aglomerados ou compensados, com detalhes em madeira natural), o que as torna pesadas.

Surgiu, então a idéia da associação entre uma prancha de leitura e/ou escrita e uma lente de aumento (lupa) que pode ser acoplada à prancha para ser apoiada e deslizar enquanto o paciente lê ou escreve. Ao se cansar, sua lupa não cairá, pois ela estará fixa ao equipamento. O material escolhido para a prancha 
foi o PVC (policloreto de vinila), nas medidas $45 \times 35 \mathrm{~cm}$, atendendo às necessidades de uso de quem precisar ler um jornal dobrado ao meio, que mede $32 \times 28 \mathrm{~cm}$, uma revista semanal do tipo Veja aberta $(40$ × $26,5 \mathrm{~cm})$ e meio caderno tipo universitário $(21 \times 28 \mathrm{~cm})$.

A lupa foi desenvolvida com uma lente asférica (apresenta um aumento do raio de curvatura do centro para a periferia aplanamento - proporcionando um melhor "aproveitamento" da imagem obtida, principalmente na periferia) de +15 dioptrias procedente da empresa internacional de origem australiana SOLA, com $62 \mathrm{~mm}$ de diâmetro. Essa lente foi envolvida por um anel de PVC.

O protótipo desenvolvido compôs-se de:

1) Prancha para apoio do objeto a ser lido ou escrito. Esta prancha apresenta perfis laterais nos quais deslizam duas peças ligadas a uma guia horizontal. A prancha apresenta, na sua parte de trás, local de encaixe de um apoio, para permitir a

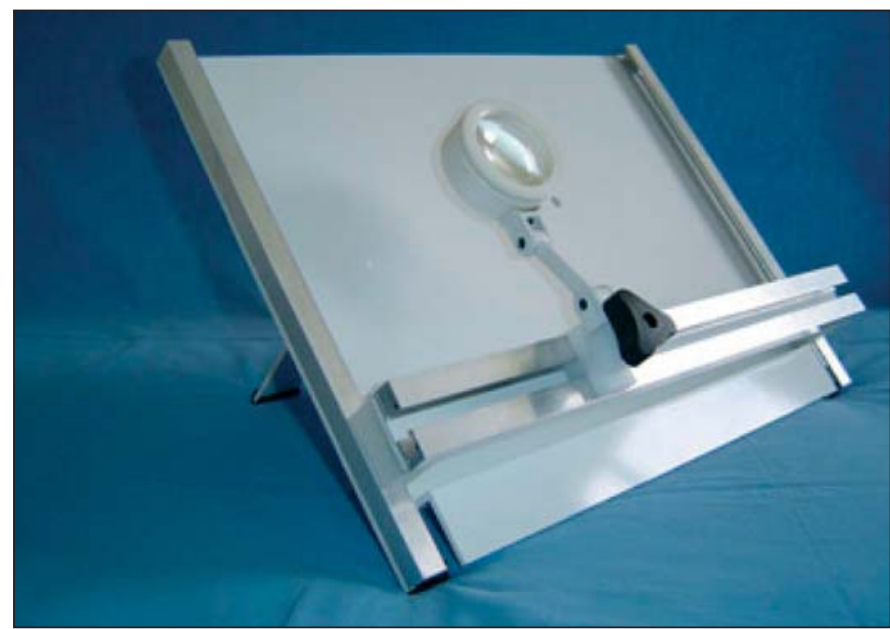

Figura 1 - Foto do protótipo em posição de uso, com a lupa orientada para cima

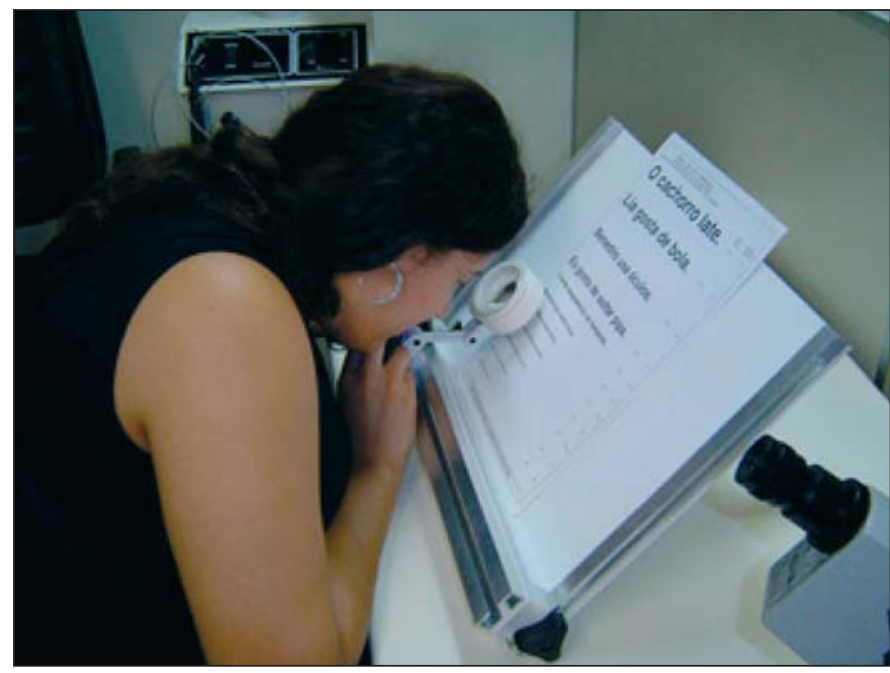

Figura 2 - Foto de paciente utilizando o equipamento sua inclinação de $45^{\circ}$ se o usuário assim o desejar e uma elevação na sua parte inferior para apoio do material de leitura e/ou escrita, com o limite máximo da espessura pré-estabelecido em $4 \mathrm{~cm}$ de lombada.

2) Guia horizontal: apresenta perfis que permitem o acoplamento de uma lupa (lente de aumento). Esta guia se encontra afastada da prancha de forma a permitir o encaixe do material de leitura e/ou escrita sobre a prancha. A guia horizontal possui, nas extremidades, peças deslizantes laterais, que se encaixam nos perfis laterais da prancha, permitindo o deslocamento vertical da guia. Essa guia horizontal poderá ser fixada, impedindo a sua movimentação vertical no ponto desejado através de um botão de rosqueamento na extremidade, que atravessa a peça deslizante lateral de tal forma que o usuário possa fazer o acompanhamento de uma linha do texto.

3) Lupa: apresenta um cabo cuja extremidade pode ser acoplada na peça que irá deslizar nos perfis da guia horizontal através de um botão de rosqueamento; seu cabo é provido de eixos que regulam a aproximação ou o afastamento da lente de aumento, podendo-se ajustar a distância focal da lente na distância adequada à espessura do material a ser lido, permitindo a acomodação de um livro com até $4 \mathrm{~cm}$ de lombada. Ao se travar a guia horizontal do equipamento com o parafuso lateral, permite-se que esta lupa pré-focada no material de leitura excursione horizontalmente de modo que, com a utilização de apenas uma das mãos para deslocamento da lupa, a pessoa possa ler uma linha do texto sem perdê-la e sem sair do foco.

A realização do protótipo foi executada pelo técnico Celso Faustino do Prado sob supervisão da autora do trabalho, com as máquinas necessárias, no LAME, Laboratório de Modelos e Ensaios da FAUUSP, com materiais adquiridos no mercado.

A escolha do material para a prancha de leitura e de seu apoio na região posterior recaiu sobre o plástico PVC (policloreto de vinila) por ser leve, de fácil limpeza e conferir um aspecto de contemporaneidade ao equipamento, além de ser resistente a quedas. A cor escolhida foi branca, pelo fato de ser neutra e não interferir com o material de leitura. A ausência de brilho excessivo também foi um fator importante a ser considerado, pois alguns pacientes com baixa visão podem apresentar dificuldades relacionadas ao reflexo da luz necessária para iluminar o material de leitura, idealmente proveniente de uma luminária, cuja posição ideal seria atrás da cabeça do paciente, segundo a literatura consultada.

A possibilidade do uso manual da lupa para o paciente transportá-la e usá-la de forma rápida, fora do contexto de uma leitura mais prolongada, agrega valor ao produto.

\section{DISCUSSÃO}

O design de cunho social vem ganhando cada vez mais espaço no mundo de hoje, na medida em que se discute a sua influência positiva na terapêutica. Dentro dessa área, soluções que contemplem a população idosa vêm ganhando cada vez mais destaque. 
Especialistas em Desenho Industrial de projeção internacional discutem que o design discrimina amplos setores da população e que são escassos os designers capazes de inventar e desenvolver a classe de produtos que verdadeiramente se necessitam ${ }^{(12)}$; é essencial a aplicação do design em campos como o desenho de instrumental médico e odontológico, equipamentos laboratoriais, equipamentos de ensino e exercício para pessoas deficientes, mutiladas e incapacitadas e o design de elementos relacionados à saúde, como camas hospitalares, macas, mesas cirúrgicas, etc ${ }^{(13)}$.

No design nacional, é preciso ressaltar o trabalho do EquipHos, o Centro de Tecnologia Hospitalar e de Engenharia de Reabilitação do Sarah (Instituto Nacional de Medicina do Aparelho Locomotor), projeto de natureza multidisciplinar, que conta com a participação do médico Aloysio Campos da Paz Jr., cirurgião-chefe e do arquiteto João Filgueiras Lima, o Lelé, que se preocupou tanto com o design de equipamentos como as "camas-maca" quanto com o projeto dos hospitais que desenvolveu. A pesquisa em bioengenharia do Instituto do Coração (Incor), do Hospital das Clínicas da USP, sob a supervisão do médico e engenheiro Adolfo Leiner, também merece destaque, pois conta com um conjunto significativo de produtos de última geração ${ }^{(14)}$. No design nacional, na Disciplina de Oftalmologia da Faculdade de Medicina da USP podese citar a participação dos seguintes médicos no desenvolvimento pioneiro no nosso meio de equipamentos e produtos da área: Prof. Dr. Newton Kara-José (estesiômetro), Prof. Dr. Hisashi Suzuki (crioextrator de catarata com seringa plástica e espiral e ponta de fio de cobre, vitreófago, diatermocoagulador bipolar ${ }^{(15)}$ e gancho de íris ${ }^{(16)}$ para dilatação mecânica da pupila), Prof. Dr. Remo Susanna Jr. (implante valvular para tratamento cirúrgico do glaucoma) e o Dr. José Américo Bonatti (crioextrator para cirurgia de catarata aperfeiçoado com caneta de polipropileno e bloco interno e ponta de cobre (patenteado), equipamento de geração e registro de pressão intra-ocular ${ }^{(17)}$, oftalmoscópio binocular indireto ${ }^{(18)}$ e videoceratoscópio $\left.{ }^{(19)}\right)$. Dentro da área de Visão Subnormal, é importante citar o desenvolvimento de sistemas telescópicos de custo reduzido apresentado na "International Conference on Low Vision" em New York, USA, 1999, projeto da equipe do Prof. Dr. Marcos W. Sampaio e da Dr ${ }^{a}$. Maria Aparecida Onuki Haddad, que utiliza tubo de plástico preto de acondicionamento de filmes fotográficos ${ }^{(20)}$; também no nosso meio merece ser mencionado, nesta mesma área, o projeto da $\mathrm{Dr}^{\mathrm{a}}$. Luciene Chaves Fernandes e do Dr. Sérgio Jacobovitz em que para feitura do sistema telescópico são utilizados tubos adaptados de $\mathrm{PVC}^{(10)}$.

Para a realização do design relacionado à saúde, é de fundamental importância a definição do problema ${ }^{(21-22)}$. Por essa razão foi estabelecida uma etapa fundamental do projeto em questão, a observação participativa dos pacientes do Serviço de Visão Subnormal da Clínica Oftalmológica do Hospital das Clínicas da Faculdade de Medicina da USP. Considero essencial a colaboração dos pacientes e da equipe de atendimento liderada pelo Prof. Dr. Marcos W. Sampaio.
Citando considerações de designers e críticos de design, como Tomás Maldonado e Gui Bonsiepe, a Prof ${ }^{\underline{q}}$. Dr ${ }^{\underline{q}}$. Maria Cecília Loschiavo dos Santos ressalta, em sua tese de LivreDocência, a dimensão pluralística e interdisciplinar do design, cuja tendência é ser enquadrado no paradigma da interdisciplinaridade e complexidade, "raiz de muitos programas de pesquisa e pós-graduação em design hoje vigentes"(23). Em sua discussão a respeito da complexidade, merece destaque a citação de Bonsiepe em seu último livro publicado em língua portuguesa, "Design do material ao digital" que questiona tanto o conteúdo quanto a orientação de desenho industrial, "tal como o conhecemos em países centrais", assinalando a existência de dois designs no mundo: "nos e para os países centrais e o design para a periferia"(24).

Esse é um produto que apresenta uma interessante relação custo/benefício para um país como o nosso, pois, recursos sofisticados e de alto custo dificilmente poderiam ser absorvidos pela maioria da população. Considerando-se a sua produção seriada, deve-se pensar além da compra para uso individual, geradora de custos para o adquirente. A adoção desse equipamento por bibliotecas públicas, escolas, centros de lazer, centros comunitários, hospitais e centros de saúde atendendo à população deficiente visual jovem e idosa diluiria o seu custo por usuário a níveis irrisórios, prestando um grande benefício à comunidade.

Uma próxima etapa a ser considerada será a realização de testes padronizados com portadores de visão subnormal utilizando o produto.

A contribuição do designer para o bem-estar humano tem sido tema de diversas pesquisas na atualidade. $\mathrm{O}$ reconhecimento das necessidades do portador de baixa visão ou de outras patologias implica a valorização desses indivíduos, contribuindo para diminuir ou quiçá findar o estigma e preconceito associados a eles.

\section{CONCLUSÕES}

Através do design, foi desenvolvido um projeto e executado um protótipo, de prancha para leitura com lente acoplada que mantém fixos a distância focal e a linha do texto a ser lido, produto este que não foi encontrado dentre os produtos pesquisados de auxílio à Visão Subnormal. Além do benefício funcional, o equipamento desenvolvido possui qualidades estéticas que podem contribuir para melhorar o estado de ânimo dos deficientes visuais e para diminuir o preconceito em relação a eles.

\section{ABSTR ACT}

Purpose: To develop the design of a near low vision aid that has esthetic and functional qualities intending to give its user a better life quality. Methods: To verify low vision people's main needs concerning the current devices for near distance; 
to identify possible design interventions to make a project that fulfills their main needs. Results: A prototype having a reading stand tied to the end of a magnifier that moves horizontally and vertically permitting the horizontal reading of a text line was developed. Conclusions: The developed device fulfills low vison people's visual and handling needs for near distance, contributing to the easiness to read and write.

Keywords: Vision, low; Equipment and supplies; Equipment design; Reading

\section{REFERÊNCIAS}

1. Camargo ML. A história dos óculos. Sinopse Oftalmol. 2000;(3):71-5.

2. Goodrich GL \& Arditi A. An Interactive History - The Low Vision Timeline. In: Stuen C, Arditi A, Horowitz A, Lang MA, Rosenthal B, Seidman K, editors. Vision rehabilitation: assessment, intervention, and outcomes. Lisse: Swets \& Zeitlinger 2000. p.3-9.

3. CID - Classificação Estatística Internacional de Doenças e Problemas Relacionados à Saúde. Doenças do olho e anexos (H00-H59). 10ª rev. São Paulo: Edusp; 2004. cap. 7.

4. Haddad MAO et al. Causes of Visual Impairment in Childhood and Adolescence: A Retrospective Study of 1917 Cases. In: Stuen C, Arditi A, Horowitz A, Lang MA, Rosenthal B, Seidman K, editors. Vision rehabilitation: assessment, intervention, and outcomes. Lisse: Swets \& Zeitlinger; 2000. p.371-5.

5. Temporini ER, Kara-José N. A perda da visão: estratégias de prevenção. Arq Bras Oftalmol. 2004;67(4):597-601.

6. Felippe C. Mudando de foco. Cresce a oferta de produtos para pessoas com visão subnormal. Univ. Visual [periódico na Internet]. 2003 [citado 2004 Jun 11]; (10):[cerca de 4 p.]. Disponível em: http://www.universovisual.com.br/ publisher/preview.php?id_mat=237

7. Plut A. Alívio para os olhos. Revista 20/20 Brasil. 2004;(16):40-2

8. ESPECIA1. Desafio: o crescimento da população idosa brasileira. Jornal do CREMESP. 2004;(198):1-8. Disponível em: http://www.cremesp.org.br/?site Acao $=$ Jornal\&id $=303$.
9. Visão Subnormal [apostilado]. In: III Curso de Reciclagem em Oftalmologia do Departamento de Oftalmologia do Hospital das Clínicas da Faculdade de Medicina da USP. São Paulo, Maio 8; 2004. São Paulo: Universidade de São Paulo; 2004.

10. Haddad MAO, Sampaio MW, Kara-José N. Auxílios para baixa visão. São Paulo: Editora Laramara; 2001.

11. Colenbrander A. Reabilitação de baixa visão. In: Veitzman S. Visão subnormal. São Paulo: Cultura Médica; 2000. p.87-109. [Coleção de Manuais Básicos].

12. Margolin V. Um "modelo social" de design: questões práticas e de pesquisa. Design em Foco. 2004;1(1):43-8.

13. Papanek VJ. Diseñar para el mundo real: ecologia humana y cambio social. Madrid: H. Blume Ediciones; 1977.

14. Santos MCL. Por um design para a vida. Design \& Interiores. 1988;1(6):54-8.

15. Suzuki H, Suzuki R, Suzuki CR. Diatermo coagulador bi-polar coaxial: modo de construção. Arq Bras Oftalmol. 1995;58(3):175-6.

16. Suzuki H, Suzuki R, Suzuki CR. Gancho de íris para dilatação mecânica da pupila: modo de construção. Arq Bras Oftalmol. 1995;58(5):367-8.

17. Bonatti JA, Suzuki H, José NK, Matheus LCA. Desenvolvimento de equipamento de geração e registro de pressão intra-ocular suportada por perfuração corneana colada com fibrina. Arq Bras Oftalmol. 1997;60(5):514-5.

18. Cavalcante I. Ataque à miopia com raio laser. Jornal da USP. 1991;5(171):12

19. Oliveira AC, Yasuoka FMM, Tonissi Jr. SA, Cansian AM, Ramos JEB, Romão AC, et al. Construção de um vídeokeratoscópio para análise topográfica da córnea [abstract]. In: XV Encontro Nacional de Física da Matéria Condensada, 1992; Caxambu. Programa e Resumos. XV Encontro Nacional de Física da Matéria Condensada; 1992.

20. Haddad MAO et al. Low Cost Telescopic System: Its Effectiveness in Cases of Macular Retinochoroiditis Due to Congenital Toxoplasmosis. In: Stuen C, Arditi A, Horowitz A, Lang MA, Rosenthal B, Seidman K, editors. Vision rehabilitation: assessment, intervention, and outcomes. Lisse: Swets \& Zeitlinger; 2000. p.195-9.

21. Dorfles G. El diseño industrial y su estética. Barcelona: Editorial Labor; c1968.

22. Munari B. Das coisas nascem coisas. São Paulo: Martins Fontes; 2002.

23. Santos MCL. Cidades de plástico e papelão: o habitat informal dos moradores de rua em São Paulo, Los Angeles e Tóquio [tese]. São Paulo: Faculdade de Arquitetura e Urbanismo. Universidade de São Paulo; 2003.

24. Bonsiepe G. Design do material ao digital. Florianópolis: CTAI/LBDI Laboratório Brasileiro de Design; 1997.

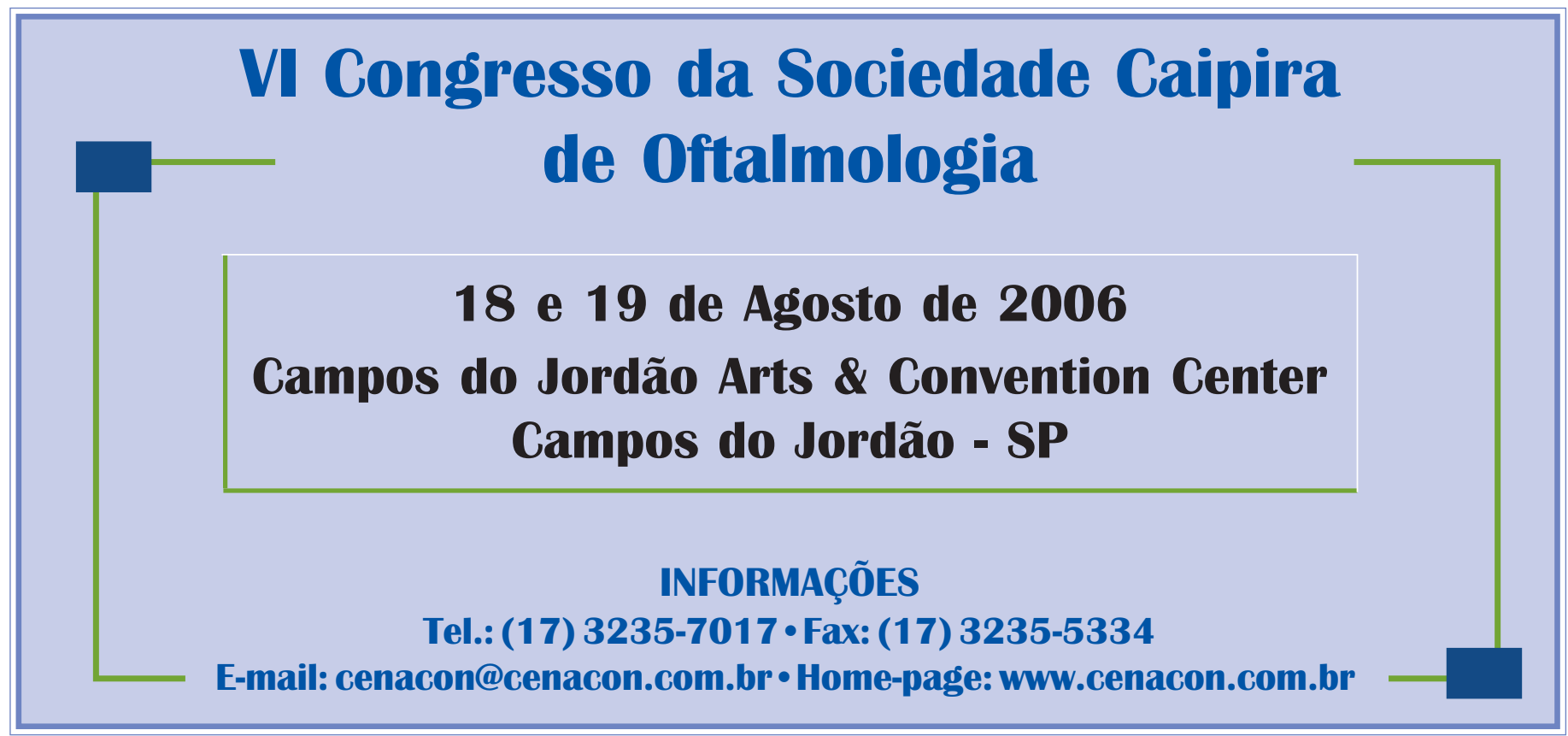

